\title{
Quantum Invariants Descending from the Joule-Lenz Law for the Dissipated Energy Applied in Calculating the Rate of Electron Transitions
}

\begin{abstract}
S. OlszeWSKI*
Institute of Physical Chemistry, Polish Academy of Sciences, M. Kasprzaka 44/52, 01-224 Warsaw, Poland

(Received January 8, 2016; in final form January 2, 2017)

The Joule-Lenz law for a classical expense of energy is transformed into a formula representing a quantummechanical invariant composed of the interval of energy connected with an electron transition and the corresponding interval of transition time between two quantum levels. Time and energy enter the invariant formula on an equal footing, moreover the time intervals converge with the time periods characteristic for the examined quantum systems. These properties imply to consider the time intervals as quanta of time having character similar to that possessed by the energy. Another result of the transformation of the Joule-Lenz law is the time rate of energy of the quantum transitions. This rate is calculated on a fully non-probabilistic way. When examined for the hydrogen atomic spectrum taken as an example, the obtained quantum rate is by many orders larger than a classical transition rate.
\end{abstract}

DOI: 10.12693/APhysPolA.131.226

PACS/topics: 03.65.-w, 03.65.Sq

\section{Introduction}

An advantage of the old quantum theory is that the motion of the electron particles entering the quantum systems is an evident property although the mechanism of that motion did not become quite clear. In course of the development of the theory, beyond the quantum property concerning a discrete separation of the energy levels, also the notion of separate time periods associated with these levels has been developed.

Both energy and time of a given system could be coupled in the formula known as a quantum invariant because of the role played by the Planck constant $h$ in this relation. The formula is a product of the double value of the average of the kinetic energy $2 E_{\mathrm{kin}}$ of a quantum system calculated over the system time period $T$ where both $E_{\text {kin }}$ and $T$ belong to the same quantum level $n$ [1]:

$$
2 \bar{E}_{\mathrm{kin}}^{(n)} T_{n}=n h .
$$

The formula (1) is often written also as [2]:

$$
\frac{2 \bar{E}_{\mathrm{kin}}^{(n)}}{\nu_{n}}=n h,
$$

where $\nu_{n}$ is the motion frequency,

$$
\nu_{n}=\frac{1}{T_{n}},
$$

applied instead of $T_{n}$.

In fact (1) is equivalent to the Bohr-Sommerfeld formula stating that the momentum $p_{n}$ of the particle possessed in a quantum state $n$ integrated over a closed path of the position variable $q_{n}$ gives

\footnotetext{
*e-mail: olsz@ichf.edu.pl
}

$$
\oint p_{n} \mathrm{~d} q_{n}=\int p_{n} \frac{\mathrm{d} q_{n}}{\mathrm{~d} t} \mathrm{~d} t=2 E_{\text {kin }}^{(n)} \int \mathrm{d} t=2 E_{\text {kin }}^{(n)} T_{n},(4)
$$

where a separation of the variable $\mathrm{d} t$ from the integrand $2 E_{\text {kin }}^{(n)}$ indicated in (4) does hold only when the kinetic energy is a constant number in course of the particle motion. Otherwise the formula (1) is valid instead of (4). The last step in (1) and (4) is a number obtained from particular quantum calculations; see Sect. 2. It can be noted that final result in (4) can be considered as corresponding with the Heisenberg commutator formula

$$
\sum_{k}\left(p_{n k} q_{k n}-q_{n k} p_{k n}\right)=\frac{h}{2 \pi \mathrm{i}} ;
$$

see Ref. [3].

\section{Invariant formulae obtained for the examples of the periodic motion}

A typical motion of Sect. 1 is the electron circulation in the hydrogen atom. For the circular Bohr orbit of the radius $r_{n}$ applied in this case we have [1]:

$$
r_{n}=\frac{n^{2} \hbar^{2}}{m e^{2}}
$$

the velocity along the orbit is

$$
v_{n}=\frac{\mathrm{d} q_{n}}{\mathrm{~d} t}=\frac{e^{2}}{n \hbar},
$$

and the time period of the circulation is

$$
T_{n}=\frac{2 \pi r_{n}}{v_{n}}=\frac{2 \pi n^{2} \hbar^{2}}{m e^{2}} \frac{n \hbar}{e^{2}}=\frac{2 \pi n^{3} \hbar^{3}}{m e^{4}} .
$$

In effect the integral in (4) becomes

$$
\begin{gathered}
\oint p_{n} \mathrm{~d} q_{n}=m \int v_{n} \frac{\mathrm{d} q_{n}}{\mathrm{~d} t} \mathrm{~d} t=m \int v_{n}^{2} \mathrm{~d} t=m v_{n}^{2} T_{n}= \\
m \frac{e^{4}}{n^{2} \hbar^{2}} \frac{2 \pi n^{3} \hbar^{3}}{m e^{4}}=2 \pi n \hbar=n h .
\end{gathered}
$$


Another example is the electron free particle in a onedimensional potential box of length $L$; see e.g. [4]. In this case the particle energy is

so

$$
E_{n}=E_{\mathrm{kin}}^{(n)}=\frac{n^{2} h^{2}}{8 m L^{2}}=\frac{1}{2} m v_{n}^{2},
$$

$$
v_{n}=\frac{n h}{2 m L}
$$

and

$$
T_{n}=\frac{2 L}{v_{n}}=\frac{4 L^{2} m}{n h} .
$$

This gives

$$
\begin{aligned}
& \oint p_{n} \mathrm{~d} q_{n}=m \int v_{n} \mathrm{~d} t=m v_{n}^{2} T_{n}=2 E_{\text {kin }}^{(n)} T_{n}= \\
& \frac{n^{2} h^{2}}{4 m L^{2}} \frac{4 L^{2} m}{n h}=n h .
\end{aligned}
$$

The case of the harmonic oscillator is slightly more complicated because the kinetic energy of the oscillator depends on $t$. We have for all states of the oscillator the velocity [5]:

$$
v=\frac{\mathrm{d} x}{\mathrm{~d} t}=v_{n}=\left(\frac{k}{m}\right)^{1 / 2}\left(a_{n}^{2}-x^{2}\right)^{1 / 2},
$$

where $x=x(t)$ and $a_{n}$ is the oscillator amplitude coupled with energy $E_{n}$ by the formula

$$
E_{n}=\frac{k a_{n}^{2}}{2}=n \hbar \omega=n \hbar\left(\frac{k}{m}\right)^{1 / 2} .
$$

$k$ is the force constant of the oscillator, $\omega$ is the circular frequency.

Since for the appropriate boundary conditions

$$
x=a_{n} \cos \omega t
$$

and

$$
\frac{\mathrm{d} x}{\mathrm{~d} t}=-a_{n} \omega \sin \omega t,
$$

we have the average value

$$
\begin{aligned}
& \overline{\left(\frac{\mathrm{d} x}{\mathrm{~d} t}\right)^{2}}=a_{n}^{2} \omega^{2} \overline{\sin ^{2} \omega t}=\frac{1}{2} a_{n}^{2} \omega^{2}, \\
& 2 \bar{E}_{\mathrm{kin}}^{(n)}=m a_{n}^{2} \omega^{2}=E_{n}
\end{aligned}
$$

and

$$
T_{n}=T=\frac{2 \pi}{\omega}
$$

are the time periods valid for any $n$. Therefore

$$
2 \bar{E}_{\mathrm{kin}}^{(n)} T_{n}=E_{n} \frac{2 \pi}{\omega}=n \hbar 2 \pi=n h .
$$

\section{Electrodynamics provides us with new invariants for the intervals of energy and time}

The basic formula (1) and its examples given in Sect. 2 concern the parameters $E_{\text {kin }}^{(n)}$ and $T_{n}$. But because of the virial theorem we have

$$
\left|E_{n}\right|=E_{\mathrm{kin}}^{(n)}
$$

$$
\bar{E}_{\mathrm{kin}}^{(n)}=\bar{E}_{\mathrm{pot}}^{(n)}=\frac{1}{2} E_{n}
$$

holds for the averaged kinetic and potential energy of the oscillator. Therefore in fact the formula (1) refers to the whole energy and whole period of time belonging to a given quantum state $n$.

But in physics we are often interested in the fractions of $E_{n}$. For example the transition energy between levels $n+1$ and $n$ in the hydrogen atom,

$$
\Delta E=E_{n+1}-E_{n},
$$

does satisfy, in general, the relation

$$
\Delta E=\alpha E_{n},
$$

where

$$
|\alpha| \neq 1
$$

for any $n$. Our aim is to demonstrate that also $\Delta E$ in (24), and the transition time $\Delta t$ corresponding to energy $\Delta E$, can enter an invariant product containing the Planck constant $h$.

To this purpose the Joule-Lenz law for the transition rate of energy $\Delta E$ within the time interval $\Delta t$ can be considered $[6,7]$ :

$$
\frac{\Delta E}{\Delta t} \approx R i^{2}
$$

$R$ is the electric resistance

$$
R=\frac{V}{i},
$$

where $V$ is a voltage and $i$ is the intensity of the electric current connected with the transition (24).

If we assume that

$$
|\Delta E| \ll\left|E_{n}\right|,\left|E_{n+1}\right|
$$

$(n \gg 1)$, the current intensities

$$
i_{n}=\frac{e}{T_{n}}, \quad i_{n+1}=\frac{e}{T_{n+1}}
$$

should be not much different each from other, so

$$
i_{n} \approx i_{n+1} \approx i=\frac{e}{T} \text {. }
$$

By putting that

$$
e V=\Delta E \text {, }
$$

we obtain from (26), (27) and (30) the relation

$$
\frac{\Delta E}{\Delta t}=\frac{\Delta E}{e} \frac{T}{e}\left(\frac{e}{T}\right)^{2}=\frac{\Delta E}{T} .
$$

This gives the result

$$
\Delta t=T \text {. }
$$

But also another result comes out from an examination of the special cases of $R$. For all quantum systems examined in Sect. 2 we obtain for large $n$ a good accuracy satisfied by the relation

$$
R=\frac{h}{e^{2}}
$$

see Sect. 4. This $R$ is well-known from the physics of the quantum Hall effect [8]. In virtue of (33) and (34) the relation in (26) becomes

or $\frac{\Delta E}{T}=\frac{h}{e^{2}} \frac{e^{2}}{T^{2}}=\frac{h}{T^{2}}$ 
$T \Delta E=h$

This is an invariant of a different nature than (1), for because of (33) - the formula (36) can be written also as (see here [9-12])

$$
\Delta t \Delta E=h \text {. }
$$

Neither $\Delta t$ nor $\Delta E$ are parameters representing the whole of a quantum state $n$. In effect of (37) we have

$$
\frac{\Delta E}{\Delta t}=\frac{(\Delta E)^{2}}{h} \text {. }
$$

\section{Invariance of the electric resistance $R$ obtained for electron transitions in different quantum systems and a check of the formula (37)}

For transitions (24) in the hydrogen atom we have

$$
\begin{aligned}
& \Delta E=E_{n+1}-E_{n}=\frac{m e^{4}}{2 \hbar^{2}}\left[-\frac{1}{(n+1)^{2}}+\frac{1}{n^{2}}\right] \\
& \cong \frac{m e^{4}}{2 \hbar^{2}} \frac{2 n}{n^{4}}=\frac{m e^{4}}{\hbar^{2} n^{3}}
\end{aligned}
$$

and

$$
T_{n+1} \approx T_{n}=T=\Delta t=\frac{2 \pi n^{3} \hbar^{3}}{m e^{4}}
$$

see (8). Because of (27), (30), and (31) we have

$$
R=\frac{\Delta E}{e} \frac{1}{e} T=\frac{m e^{4}}{\hbar^{2} n^{3}} \frac{1}{e^{2}} \frac{2 \pi n^{3} \hbar^{3}}{m e^{4}}=\frac{2 \pi \hbar}{e^{2}}=\frac{h}{e^{2}},
$$

which is a quantity equal to (34). The check of the formula (37) gives

$$
\Delta t \Delta E=T \Delta E=\frac{2 \pi n^{3} \hbar^{3}}{m e^{4}} \frac{m e^{4}}{n^{3} \hbar^{3}}=2 \pi \hbar=h .
$$

A similar examination of the free-electron motion in a potential box of length $L$ gives, in the first step,

$$
\Delta E=E_{n+1}-E_{n}=\frac{(n+1)^{2}-n^{2}}{8 m L^{2}} h^{2} \cong \frac{n h^{2}}{4 m L^{2}} .
$$

When $\Delta E$ from (42) is coupled with the time period

$$
T_{n+1} \approx T_{n}=T=\Delta t=\frac{4 L^{2} m}{n h}
$$

given in (12) they give the resistance

$$
R=\frac{\Delta E}{e} \frac{T}{e}=\frac{n h^{2}}{4 m L^{2}} \frac{1}{e^{2}} \frac{4 L^{2} m}{n h}=\frac{h}{e^{2}} .
$$

The $\Delta t=T_{n}$ in (43) combined with $\Delta E$ in (42) gives

$$
\Delta t \Delta E=T \Delta E=\frac{4 L^{2} m}{n h} \frac{n h^{2}}{4 m L^{2}}=h .
$$

The oscillator case is represented by$$
\Delta E=(n+1-n) \hbar \omega=\hbar \omega=h \frac{1}{T},
$$

$$
T=\frac{h}{\Delta E} .
$$

Therefore

$$
R=\frac{\Delta E}{e} \cdot \frac{T}{e}=\frac{h}{e^{2}}
$$

because of (47). Evidently from (33) and (47):

$$
T \Delta E=\Delta t \Delta E=h \text {. }
$$

A characteristic point is that no $R$ has a net dependence on $n$.

\section{Quantum aspects of the Joule-Lenz law for the dissipated energy applied to the atomic spectra}

The main advantage of the quantum representation of the Joule-Lenz theory seems to be an approach to the time interval $\Delta t$ connected with the electron transition between the neighbouring levels $n+1$ and $n$; the levels are separated by the energy interval $\Delta E$ in (24). The relation between $\Delta t$ and $\Delta E$ is given in (49).

This relation can be extended succesively to $\Delta t$ between the levels $n+q$ and $n$, where $q>1$, therefore the separation between the levels can be larger than $\Delta E$ in (49). In consequence the Joule-Lenz approach can be used in principle to calculate the intensity of the whole atomic spectrum. This kind of approach seems to be more advantageous than a conventional probabilistic theory of quantum transitions because it avoids a complicated calculation imposed by the use of the electron wave functions of the atom and the matrix elements based on them.

In considering the emission spectra we assume that the excited states of the atom are approximately in thermal equilibrium, hence the number of states in any given atom is proportional to the Boltzmann factor [13]. In effect the intensity of the spectral line - which in the present theory is defined by the ratio of the energy interval $\Delta E$ to the time interval $\Delta t-$ can be considered as proportional to the transition probability obtained from the quantum-mechanical formalism [13]. In the papers $[14,15]$ this idea has been applied to the intensity spectrum of the atomic hydrogen for which the transitions between the states

$$
\begin{aligned}
& n^{\prime} p \rightarrow n s, \\
& n^{\prime} d \rightarrow n p, \\
& n^{\prime} f \rightarrow n d, \\
& n^{\prime} g \rightarrow n f,
\end{aligned}
$$

have been examined. Here rather low quantum numbers $n^{\prime}$ and $n$ satisfying the relation

$$
n^{\prime}>n
$$

were taken into account.

In the probabilistic approach the emission intensity of energy in course of an electron transition is proportional to (i) an unknown number of electron carriers involved in the transition, (ii) the transition energy, and (iii) the quantum-mechanical probability of transition obtained from the wave functions describing the energy levels $[16,17]$. In order to eliminate the problem of proportionality coefficients, a pattern of the ratios of semiclassical intensities and that of the ratios of quantummechanical probabilities corresponding to different pairs of the electron transitions is considered in [14, 15].

In general, the pattern of the ratios of semiclassical emission intensities fits better than those obtained from the ratios of quantum-mechanical probabilities of transitions. 
Evidently the semiclassical formalism does not provide us with the selection rules between different quantum states, for example the rules concerning states having a different quantum number $l$ of the angular momentum. In the formulae (50)-(54) only transitions from state $l^{\prime}$ to state $l$, where $l^{\prime}>l$, are considered. But in spectroscopy the emission in an opposite situation concerning $l^{\prime}$ and $l$, namely from the state $l^{\prime}$ to state $l$ where $l^{\prime}<l$ is also possible. Such transitions have been examined in [15]. In this case, however, the results for the semiclassical intensity ratios and ratios of the quantum-mechanical transition probabilities occur to be of a poorer agreement than obtained for transitions represented by (50)-(54).

Other problems concerning the quantum aspects of the Joule-Lenz law have been studied in $[18,19]$.

In Ref. [19] a semiclassical approach to the time of the electron transition between two quantum levels is studied.

\section{Quantum transition rate compared with a classical rate}

Both $\Delta E$ and $\Delta t=T$ can depend on the boundary conditions. Evidently the electron transition energy between two quantum levels should not necessarily provide us with the energy of a photon. A decisive point is here the size of $T=T_{n}$. For example from (12) the time

$$
T_{n}=T_{1}=\frac{4 L^{2} m}{h}
$$

gives the wavelength of a visible light, say $\lambda=c T_{1}=$ $5 \times 10^{-5} \mathrm{~cm}$, on condition

$$
(2 L)^{2} \cong 10^{-14} \mathrm{~cm}^{2}
$$

or $2 L \approx 10^{-7} \mathrm{~cm}$.

The quantum transition rate can be compared with a classical rate for the hydrogen atomic spectrum taken as an example. The classical rate is based on the formula $[20,21]$ :

$$
\left|\frac{\mathrm{d} E}{\mathrm{~d} t}\right|=\frac{\Delta E}{\Delta t}=\frac{(2 \pi \nu)^{3}}{3 c^{3}} \boldsymbol{P}^{2} .
$$

Here $\nu$ is the frequency of the electromagnetic wave connected with the electron transition, and

$$
|\boldsymbol{P}|=e \Delta r
$$

is the polarization amplitude induced by a dipole moment associated with transition from the atomic level $n+1$ to level $n$.

We expect that quantum transition rate given in (37a) is much more rapid than a classical one, so

$$
\frac{(\Delta E)^{2}}{h}>\frac{(2 \pi \nu)^{4}}{3 c^{3}} \boldsymbol{P}^{2}=\frac{(2 \pi)^{4}(\Delta E)^{4}}{3 c^{3} h^{4}}(e \Delta r)^{2} .
$$

In fact a transformation of the formula (57) gives in view of (6) and (38):

$$
\begin{gathered}
1>\frac{(2 \pi)^{4}}{3 c^{3} h^{3}}(\Delta E)^{2} e^{2}\left[\frac{(n+1)^{2}-n^{2}}{m e^{2}} \hbar^{2}\right]^{2} \cong \\
\frac{(2 \pi)^{4}}{3 c^{3} h^{3}}\left(\frac{m e^{4}}{\hbar^{2} n^{3}}\right)^{2}\left(\frac{2 n \hbar^{2}}{m e}\right)^{2}=\frac{e^{6}}{\hbar^{3} c^{3}} \frac{8 \pi}{3} \frac{1}{n^{4}}=\left(\frac{e^{2}}{\hbar c}\right)^{3} \frac{8 \pi}{3 n^{4}} .
\end{gathered}
$$

Because of

$$
\frac{e^{2}}{\hbar c} \cong 1 / 137
$$

we obtain that a quantum rate is by more than one million times stronger than a classical one already for small $n$; for larger $n$ a predominance of the quantum speed of transition over classical one can be still stronger.

\section{Reference of the Einstein coefficients of emission to the formula (37a)}

A well-known result of the Einstein emission formula $[22,23]$ is that it is a combination of a spontaneous and induced emission represented, respectively, by the transition probabilities $A_{n+1, n}$ and $B_{n+1, n}$. In the Van Vleck formulation [24] the total emitted energy $\Delta E$ within the time interval $\Delta t$ is

$$
\Delta E=h \nu\left(A_{n+1, n}+B_{n+1, n} \varrho\right) \Delta t
$$

$\varrho$ is the radiation density. Here we assume that only a single electron state $n+1$ is submitted to emission of the energy

$$
\Delta E=E_{n+1}-E_{n}=h \nu
$$

in course of transition to state $n$ within the time interval $\Delta t$. In this formulation of the problem the number of the emitted quanta is $N_{r}=1$ and the emission rate is

$$
\frac{\Delta E}{\Delta t}=h \nu\left(A_{n+1, n}+B_{n+1, n} \varrho\right) \text {. }
$$

Because of (37a) and (61) the following equation instead of (62) is obtained:

$$
\frac{(\Delta E)^{2}}{h}=\Delta E\left(A_{n+1, n}+B_{n+1, n} \varrho\right)
$$

$$
\frac{\Delta E}{h}-A_{n+1, n}=B_{n+1, n} \varrho .
$$

This gives

$$
\varrho=\left(\frac{\Delta E}{h}-A_{n+1, n}\right) \frac{1}{B_{n+1, n}} .
$$

By requiring that terms $\Delta E, B_{n+1, n}$ and $\varrho$ are positive numbers, we obtain from (64) and (65) a condition which has to be satisfied in the emission process

$$
\frac{\Delta E}{h}-A_{n+1, n}=\nu-\frac{16 \pi^{4} e^{2} \nu^{3}}{3 h c^{3}} D^{2}>0,
$$

from which

$$
\nu>\frac{16 \pi^{4} e^{2} \nu^{3}}{3 h c^{3}} \frac{h}{2 \pi^{2} \nu m}=\frac{8 \pi^{2}}{3} \frac{e^{2} \nu^{2}}{c^{3} m}=A_{n+1, n} .
$$

The data for $A_{n+1, n}$ and the amplitude square of the oscillator

$$
D^{2}=\frac{h}{2 \pi^{2} \nu m}
$$

are taken from [24]. In (68) we assumed that $\nu$ represents the frequency of a single quantum $\Delta E=h \nu$ of the oscillator and not many quanta $\Delta E=n h \nu$ as it is in [24].

The formula for the limiting reciprocal value of $\nu$ obtained in (66) and (67) corresponds to the smallest admissible oscillation time period $T_{\min }$ : 


$$
\frac{1}{\nu_{\max }}=T_{\min }=\frac{8 \pi^{2} e^{2}}{3 c^{3} m} .
$$

In fact, the same $T_{\min }$ has been obtained a time ago in the Kramers-Heisenberg search on dispersion properties of radiation by atoms $[20,23]$. There was introduced the decay time $\tau_{\nu}$ of the classically oscillating electron with frequency $\nu$ :

$$
\tau_{\nu}=\frac{3 c^{3} m}{8 \pi^{2} e^{2} \nu^{2}} .
$$

Let us assume that $\nu$ is so large that its reciprocal value attains the size of the decay time $\tau_{\nu}$ given in (70) so

$$
\tau_{\nu}=\frac{1}{\nu} \text {. }
$$

The relation (71) substituted into (70) gives the following equation for $\tau_{\nu}$ :

$$
\tau_{\nu}=\frac{3 c^{3} m \tau_{\nu}^{2}}{8 \pi^{2} e^{2}}
$$

The solution of $(72)$ gives

$$
\frac{1}{\tau_{\nu}}=\frac{3 c^{3} m}{8 \pi^{2} e^{2}}
$$

which is equal to $\nu_{\max }$ obtained in (69). This parameter is useful in representing $A_{n+1, n}$ [see (67)]:

$$
A_{n+1, n}=\frac{8 \pi^{2}}{3} \frac{e^{2} \nu^{2}}{c^{3} m}=\frac{\nu^{2}}{\nu_{\max }} .
$$

Since in view of (64), (61), and (74) we have

$$
B_{n+1, n} \varrho=\nu-\frac{\nu^{2}}{\nu_{\max }},
$$

a full combination of the parameters defining the emission becomes

$$
A_{n+1, n}+B_{n+1, n} \varrho=\frac{\nu^{2}}{\nu_{\max }}+\nu-\frac{\nu^{2}}{\nu_{\max }}=\nu
$$

in accordance with the formula presented in (64).

We found that the coefficient $A_{n, n+1}$ of the spontaneous emission increases parabolically with $\nu$ to the value equal to $\nu_{\max }[$ see $(74)]$, whereas the term representing the induced emission given in (75) is roughly proportional to $\nu$ at small $\nu$, attains a maximum at

$$
\nu=\frac{1}{2} \nu_{\max },
$$

but next tends to zero when $\nu$ approaches $\nu_{\max }$.

A similar situation concerns $\varrho$ alone on condition we assume that [22]:

$$
\frac{A_{n+1, n}}{B_{n+1, n}}=\beta \nu^{3}
$$

$\beta$ is a constant. In this case [see (74)]:

$$
B_{n+1, n}=\frac{\nu^{2}}{\nu_{\max }} \frac{1}{\beta \nu^{3}}=\frac{1}{\beta \nu_{\max } \nu},
$$

so - because of $(75)$ - we have

$$
\begin{aligned}
& \varrho=\frac{\nu-\frac{\nu^{2}}{\nu_{\max }}}{B_{n+1, n}}=\left(\nu-\frac{\nu^{2}}{\nu_{\max }}\right) \beta \nu_{\max } \nu= \\
& \beta\left(\nu_{\max } \nu^{2}-\nu^{3}\right) .
\end{aligned}
$$

Evidently $\varrho$ vanishes both at $\nu=0$ and $\nu=\nu_{\max }$. A maximum of $\varrho$ is attained at

$$
\nu=(2 / 3) \nu_{\max }
$$

and amounts to

$$
\varrho_{\max }=\beta \frac{4}{27} \nu_{\max }^{3} .
$$

\section{Summary}

The invariance property concerning the product of the double kinetic energy and the period of time belonging to a quantum state $n$ (the Bohr-Sommerfeld quantum condition) has been extended to the case where the energy factor can be solely a fraction of the energy of a quantum state represented by the amount of energy involved in a quantum transition. Also the interval of time entering the mentioned product is that connected with transition time.

The formalism based on a classical Joule-Lenz law describing the dissipation energy has been developed in two steps. First the law is applied to the case when the transition energy, equal to the dissipation energy, is assumed to be represented by the voltage amount multiplied by the electron charge $e$; see (31). This gives the formula

$$
\frac{\Delta E}{\Delta t}=R i^{2}=\frac{\Delta E}{e} \frac{T}{e}\left(\frac{e}{T}\right)^{2}=\frac{\Delta E}{T},
$$

from which we obtain that

$$
\Delta t=T \text {. }
$$

In the next step the electric resistance $R$ associated with different cases of the electron transitions and their energy $\Delta E[$ see (24)] is separately examined. One obtains invariably the relation

$$
R=\frac{\Delta E}{e} \frac{T}{e}=\frac{h}{e^{2}}
$$

for each examined case. Formula (84) combined with (83a) provides us with equations

$$
\frac{\Delta E}{\Delta t}=\frac{h}{e^{2}} \frac{e^{2}}{T^{2}}=\frac{h}{T^{2}}
$$

and, in the next step,

$$
\Delta E T=\Delta E \Delta t=h \text {. }
$$

Because $\Delta E$ is a defined quantity, Eq. (85a) allows us to calculate $\Delta t$ in a fully deterministic way. Consequently the same property concerns the transition rate for energy which becomes also

$$
\frac{\Delta E}{\Delta t}=\frac{(\Delta E)^{2}}{h}
$$

see Eq. $(37 \mathrm{a})$

The $T$ in (85a) which satisfies (33) is a period of time belonging to one of the quantum states involved in transition; see (30). The validity of the formula (86) has been checked elsewhere - see Sect. 5 - by examining the quantum-mechanical intensity of the spectrum of the hydrogen atom taken as an example.

A non-probabilistic character of the formula (86) is a new element in the theory of quantum transitions since till present - beginning with the results attained by Planck [25] and Einstein [22] — any treatment of the spectrum intensity was referred to statistical background of that theory; see e.g. [23]. 
In fact, the interval $\Delta t$ of transitions between two quantum levels seems to be never adequately examined for an individual transition, neither in the old quantum theory, nor in quantum mechanics. The formulae (37) and (85a) indicate that this time interval behaves, in course of an electron transition, in the same way as energy. Since we speak on the quanta of energy defined by the intervals

$$
\Delta E=h \nu=h T^{-1}=h(\Delta t)^{-1},
$$

the same can be said on the intervals $\Delta t$ in connection with the notion of time: these intervals are quanta of time

$$
\Delta t=\frac{h}{\Delta E}=T
$$

identical with the time periods possessed by a quantum system submitted to transition, because of the formulae obtained in (33) and (37).

\section{References}

[1] A. Sommerfeld, Atombau und Spektrallinien, Vol. 1, 5th ed., Braunschweig, 1931 (in German).

[2] P. Ehrenfest, Ann. Phys. 341, 91 (1911), Phys. Zeitschr. 15, 657 (1914), Ann. Phys. 356, 327 (1916).

[3] M. Born, P. Jordan, Zeitsch. Phys. 34, 858 (1925).

[4] H. Eyring, J. Walter, G.E. Kimball, Quantum Chemistry, Wiley, New York 1957.

[5] A. Sommerfeld, Mechanik, Akademische Verlagsgesellschaft, Leipzig 1943 (in German).

[6] H. Lass, Vector and Tensor Analysis, McGraw-Hill, New York 1950.
[7] A.N. Matveev, Electrodynamics and the Theory of Relativity, Izd. Wyzszaja Szkola, Moscow 1964 (in Russian).

[8] A.H. MacDonald, Quantum Hall Effect. A Perspective, Kluwer, Milano 1989.

[9] S. Olszewski, J. Mod. Phys. 6, 1277 (2015).

[10] S. Olszewski, Quant. Matter 5, 664 (2016).

[11] S. Olszewski, Rev. Theor. Sci. 4, 336 (2016).

[12] S. Olszewski, J. Mod. Phys. 7, 162 (2016).

[13] H.G. Kuhn, Atomic Spectra, Academic Press, New York 1962.

[14] S. Olszewski, J. Mod. Phys. 7, 827 (2016).

[15] S. Olszewski, J. Mod. Phys. 7, 1004 (2016); J. Mod. Phys. 7, 2314 (2016).

[16] H. Bethe, "Quantenmechanik der Ein- und ZweiElektronen Probleme", in: Handbuch der Physik, Eds. H. Geiger, K. Scheel, Vol. 24, Part 1, Springer, Berlin 1933 (in German).

[17] E.U. Condon, G.H. Shortley, The Theory of Atomic Spectra, Cambridge University Press, Cambridge UK 1970.

[18] S. Olszewski, J. Mod. Phys. 7, 1440 (2016).

[19] S. Olszewski, J. Mod. Phys. 7, 1725 (2016).

[20] H.A. Kramers, W. Heisenberg, Zeits. Phys. 31, 681 (1925).

[21] W. Kuhn, Zeitsch. Phys. 33, 408 (1925).

[22] A. Einstein, Phys. Zeitsch. 18, 121 (1917).

[23] B.L. Van der Waerden, Sources of Quantum Mechanics, Dover, New York 1968.

[24] J.H. Van Vleck, Phys. Rev. 24, 330 (1924).

[25] M. Planck, Acht Vorlesungen ueber Theoretische Physik, S. Hirzel, Leipzig 1910 (in German). 